\title{
Examining Neural Reactivity to Gambling Cues in the Age of Online Betting
}

\author{
Damien Brevers $^{1} \cdot$ Guillaume Sescousse $^{2} \cdot$ Pierre Maurage $^{3} \cdot$ Joël Billieux $^{1,4}$ \\ Published online: 23 May 2019 \\ (C) Springer Nature Switzerland AG 2019
}

\begin{abstract}
Purpose of Review The goal of this review is to provide new insights as to how and why functional magnetic resonance imaging (fMRI) research on gambling cue reactivity can contribute to significant progress toward the understanding of gambling disorder. After having offered a detailed description of experimental paradigms and a comprehensive summary of findings related to gambling cue reactivity, the present review suggests methodological avenues for future research.

Recent Findings The fMRI literature on problem gambling has identified the main neural pathways associated with reactivity to gambling cues. Yet, the current knowledge on the key factors underlying cue reactivity in gambling is still very incomplete. Here, we suggest that the recent expansion of online sports betting calls for a new line of research offering a fine-grained and up-to-date approach of neural cue reactivity in gambling disorder.

Summary Experimental designs that investigate individual-specific and study-specific factors related to sports betting have the potential to foster progress toward efficient treatment and prevention of gambling disorder.
\end{abstract}

Keywords $\mathrm{fMRI} \cdot$ Cue reactivity $\cdot$ Addiction $\cdot$ Gambling disorder $\cdot$ Sports betting

\section{Introduction}

Gambling is on the rise $[1,2]$. Not only is it possible to engage in such activity at any time and place, but the omnipresence of gambling-related cues (e.g., advertising, cellphone notifications, witnessing others' thrilling experiences) constantly promotes gambling temptations [3-6]. With this around-the-clock availability and omni-

This article is part of the Topical Collection on Impulse Control Disorders

Damien Brevers

damien.brevers@uni.lu

1 Addictive and Compulsive Behaviours Lab (ACB-Lab), Health and Behaviour Institute, University of Luxembourg,

Esch-sur-Alzette, Luxembourg

2 Lyon Neuroscience Research Center - INSERM U1028 - CNRS UMR5292, PSYR2 Team, University of Lyon, Lyon, France

3 Laboratory for Experimental Psychopathology (LEP), Psychological Science Research Institute, UCLouvain, Louvain-la-Neuve, Belgium

4 Centre for Excessive Gambling, Lausanne University Hospitals (CHUV), Lausanne, Switzerland presence of cues, the evolving landscape of (online) gambling and betting raises important public health questions (e.g. [2, 7, 8•]).

Neural reactivity to addiction-related cues, as assessed with functional magnetic resonance imaging (fMRI), has repeatedly been identified as a key biomarker of disorder severity, treatment outcome, and relapse risk in cocaine, alcohol, and nicotine use disorder (e.g., [9, 10]). In this paper, we propose that in a context where online gambling opportunities are blooming, research on gambling cue reactivity could also offer a fertile ground to advance current knowledge about the cognitive and motivational determinants of gambling disorder. To reach this objective, we first present an overview of the learning processes and brain pathways underlying cue reactivity. Then, we comprehensively describe the main cue reactivity paradigms traditionally used in fMRI studies to examine cue reactivity in problem gamblers, before detailing findings from these imaging studies. Finally, by taking into account the main strengths and weaknesses of past methods and findings and capitalizing on previous model-based reviews on neural cue reactivity in substance use disorder [9-12], we identify and characterize the factors that should guide future directions for fMRI studies on gambling cue reactivity. 


\section{Processes Underlying Cue Reactivity}

Increased reactivity to addiction-related cues is assumed to result from the activation of specific associative pathways in long-term memory [13]. These associations are built and strengthened gradually through classical conditioning processes, that is, by the learning history of temporal or spatial coactivation between external (e.g., environmental cue) or internal (e.g., affective state) cues and reward consumption effects $[14,15]$. In line with this account, the incentive sensitization theory predicts that the repeated pairing of environmental cues with substance consumption leads these cues to acquire increased salience and to capture attention, over and above primary natural rewards (e.g., food, sex; [16-18]). At the cerebral level, a wealth of fMRI studies has shown that the incentive salience of substance-related cues (triggering socalled "wanting") is generated by a large and distributed brain system involving the ventral and dorsal striatum, amygdala, hippocampus, insula, anterior cingulate cortex, orbitofrontal cortex (OFC), and dorsolateral prefrontal cortex (DLPFC), as well as sensory, visual, and motor cortices [9-12].

This extended brain pathway likely reflects the complex nature of the interactions between the so-called bottom-up impulsive and top-down reflective systems. Specifically, the
fMRI literature on cue reactivity suggests that the motivational salience carried by substance-related cues may (i) sensitize or exacerbate the activity of the amygdala-striatal "impulsive" system, which generates positive affective associations and fast approach behavior toward addiction-related stimuli and (ii) subvert attention, reasoning, planning, and decisionmaking resources of the prefrontal "reflective" system to seek and reach rewards. Importantly, substance cue reactivity does not necessarily lead to weaker or hypoactive cognitive control (as commonly described by dual-process models of addictions; [14, 19, 20]), but instead redirects attention and executive control resources toward goals related to substance consumption [21-24]. In line with this account, triadic models of addiction advance that, under certain circumstances (e.g., homeostatic imbalance, reward deprivation, stress, sleep deprivation), the insular cortex plays a pivotal role in promoting the drive and motivation to get a reward by "hijacking" goal-oriented processes toward addiction-related cues at the expense of inhibitory control resources [25-35]. Taking into account these dynamic patterns of neural cue reactivity is of critical importance for studies that aim at modulating brain processes to decrease subjective states of craving. For instance, Hayashi et al. [36] highlighted that the strong

Table 1 Overview of fMRI studies on gambling cue reactivity

\begin{tabular}{|c|c|c|c|c|c|}
\hline Study & Sample & Task & Tesla & Software & Statistical threshold \\
\hline $\begin{array}{l}\text { Potenza et al. [40, 41]; } \\
\text { Balodis et al. [42] }\end{array}$ & $10 \mathrm{PrG} ; 11 \mathrm{HC} ;(9 \mathrm{CD} ; 6 \mathrm{HC})^{*}$ & $\begin{array}{l}\text { Exposure to gambling, sad } \\
\text { and happy videos }\end{array}$ & $1.5 \mathrm{~T}$ & Yale & Uncorr; whole brain \\
\hline Crockford et al. [43] & $10 \mathrm{PrG} ; 10 \mathrm{HC}$ & $\begin{array}{l}\text { Exposure to nature and } \\
\text { gambling videos }\end{array}$ & $3.0 \mathrm{~T}$ & Stimulate & Corr; whole brain \\
\hline Goudriaan et al. [44] & $\begin{array}{l}17 \text { non-smoking PrG } \\
\text { under treatment; } 18 \mathrm{HS} ; 17 \mathrm{HC}\end{array}$ & $\begin{array}{l}\text { Exposure to gambling, } \\
\text { smoking and neutral } \\
\text { pictures }\end{array}$ & $3.0 \mathrm{~T}$ & SPM2 & FWE; whole brain \\
\hline Balodis et al. [45] & $14 \mathrm{PrG} ; 14 \mathrm{HC}$ & $\begin{array}{l}\text { Monetary incentive delay } \\
\text { task }\end{array}$ & $3.0 \mathrm{~T}$ & SPM5 & FWE; ROI \\
\hline van Holst et al. [46, 47] & $16 \mathrm{PrG}$ under treatment; $15 \mathrm{HC}$ & $\begin{array}{l}\text { Go/No go task featuring } \\
\text { neutral, positive, } \\
\text { negative and gambling } \\
\text { pictures }\end{array}$ & $3.0 \mathrm{~T}$ & SPM5 & FWE; ROI \\
\hline van Holst et al. [48] & $15 \mathrm{PrG}$ under treatment; $16 \mathrm{HC}$ & $\begin{array}{l}\text { Guessing task, involving } \\
\text { monetary reward }\end{array}$ & $3.0 \mathrm{~T}$ & SPM5 & FWE; ROI \\
\hline Sescousse et al. [49] & $20 \mathrm{PrG} ; 20 \mathrm{HC}$ & $\begin{array}{l}\text { Incentive delay task, } \\
\text { involving monetary } \\
\text { reward, sexual reward, } \\
\text { and neutral trials }\end{array}$ & $1.5 \mathrm{~T}$ & SPM2 & FWE; whole brain, ROI \\
\hline Kober et al. [50] & $30 \mathrm{CD} ; 28 \mathrm{PrG} ; 45 \mathrm{HC}$ & $\begin{array}{l}\text { Exposure to gambling, sad } \\
\text { and happy videos }\end{array}$ & $3.0 \mathrm{~T}$ & SPM5 & FWE; whole brain \\
\hline Brevers et al. [51] & 14 frequent gamblers; $14 \mathrm{HC}$ & $\begin{array}{l}\text { Stop-signal paradigm } \\
\text { featuring gambling and } \\
\text { neutral pictures }\end{array}$ & $3.0 \mathrm{~T}$ & FSL/Randomize & TFCE; whole brain \\
\hline Limbrick-Oldfield [52] & $19 \mathrm{PrG}$ under treatment; $19 \mathrm{HC}$ & $\begin{array}{l}\text { Exposure to gambling, } \\
\text { food and neutral pictures }\end{array}$ & $3.0 \mathrm{~T}$ & FSL/Flame & FWE; whole brain, ROI \\
\hline
\end{tabular}

Notes. Studies between brackets present supplementary analyses and findings from a previously published database. $C D$ cocaine dependent, Corr corrected for multiple comparisons, FSL FMRIB Software Library, $F W E$ family-wise error, $H C$ healthy control, $H S$ heavy smokers, $P r G$ problem gamblers, ROI region of interest, SPM statistical parametric mapping, uncorr uncorrected for multiple comparisons TFCE Threshold-free cluster enhancement. *only in Potenza [41] 
Box 1 A chronological synthesis of the fMRI literature on neural cue reactivity to gambling cues

- 2003: Potenza et al. [40]. fMRI was used to examine the brain correlates triggered by the viewing of videos related to gambling, sad or happy scenarios in a sample of PrGs and a group of healthy controls (HCs). The scenarios were being played by actors talking directly to the camera to increase participants' immersive experience. Another strength of Potenza et al. [40] design is that each type of video was divided into three temporal epochs of interest. Specifically, the gambling video started with an individual describing a stressful situation, such as problem at work or at home (i.e., first epoch of interest). Then, the video depicted the individual driving to and entering a casino, obtaining chips, going to a table, emphasizing the excitement (i.e., second epoch of interest). Lastly, the gambling video described the "rush" and "thrill" triggered by the action of gambling (i.e., third epoch of interest). The sad and happy videos were made so that it progressively increased emotional responses of the participants, with epochs of interest corresponding to either the initial, middle and final period of viewing.

This procedure allowed Potenza et al. [40] to observe different patterns of brain activation between PrGs and HCs across the temporal epochs. Specifically, during the initial period of viewing of the gambling scenarios, PrGs displayed decreased brain activity in the lateral orbitofrontal cortex, the caudate nucleus, basal ganglia and thalamus. No difference was observed for the happy and sad videos. By contrast, during the middle period of viewing, PrGs exhibited increased activity within the cuneus and middle occipital gyrus for the gambling videos, and within the VS and posterior OFC for the happy videos. In the final period of viewing, PrGs displayed decreased activity within the for the gambling videos and within the superior frontal gyrus for the sad videos.

As a whole, findings from Potenza et al. [40] suggest that patterns of neural cue reactivity in PrGs differ according to the temporal dynamics of the action of gambling and of an emotional response. However, one main limitation of these reported findings is that contrast images were computed using uncorrected thresholds (ranging from $p<.001$ to $p<.01$ ) across the whole brain. Besides, within-group activity maps were not reported. Specifically, within-group activity maps were only used to check and elucidate the nature of the between-group differences, and were examined at a threshold of $p<0.05$.

- 2005: Crockford et al. [43]. A group of PrGs and a group of HCs viewed nature and gambling-related videos. Three different runs of visual gambling cues were selected and divided into four 30-second segments. The first video run (Casino Gambling Run) displayed individuals gambling in casino settings playing blackjack, craps, roulette, and slot machines/ VLT and receiving cash payouts. The second run (Gambling Venues Run) displayed four 30-second segments of gambling venues involving the exteriors of Las Vegas casinos. The third run (VLT Run) displayed four novel 30 -second segments of a VLT being played where viewers could observe the strategies being used. The nature video was also divided into 30second segments and consisted of wildlife and nature scenes. Subjects' craving for gambling was assessed via a 7 -points Likert scale $(0=$ absent, 7 = maximal) prior to the imaging session and at the end of each run. Importantly, participants were informed that they would have the opportunity to gamble (i.e., play a slot machine game) within the scanner and after all video sequences.

Crockford et al. [43] observed that mean change in subjective craving was significantly in PrGs than in HCs. At the brain level, within-group analyses revealed that PrGs and HCs showed significant activity in several overlapping regions in response to the gambling stimuli, including right inferior and medial frontal gyrus, and bilateral (pre)cuneus, parietal lobule, medial/inferior occipital gyrus, lingual gyrus, and fusiform gyrus. Between-group analyses revealed that PrGs exhibited higher activation in the right medial frontal gyrus, the right inferior frontal gyrus, the right parahippocampal gyrus and left fusiform gyrus. In addition, post-hoc analyses revealed that PrG participants exhibited increased activation in the dorsal visual processing stream (bilateral precuneus and right inferior parietal lobule) in response to viewing a VLT video, whereas HCs activated the ventral visual processing stream (right medial occipital gyrus, right cuneus, right lingual gyrus and left lingual gyrus) when viewing the video depicting gambling venues. No correlation analyses were reported between craving scores and brain imaging data.

- 2008: Potenza et al. [41]. This review paper included supplementary brain imaging analyses on data from past studies from Potenza et al. [41] in PrGs, from Wexler et al. [54] in CDs during gambling or drug video scenarios, respectively, and for the entire period of viewing of the addiction videotapes. These analyses showed increased activity within ACC while viewing addiction-related videos in CDs, as compared to PrGs. Nevertheless, similarly as in Potenza et al. [41], the low sample size (PrG: $n=10$; HC: $n=11$ ) and the uncorrected thresholds of brain imaging analyses the statistical validity of these findings.

- 2010: Goudriaan et al. [44]. This study used a cue-reactivity task where participants were required to press a response button with their left index finger when a face was present in the picture and had to press a response button with their right index finger when no face was present. There was no time limit to answer. The pictures were either neutral, smoking-related or gambling-related. In addition, low-level baseline pictures with arrows pointing to the left or right were presented, and a left or right response had to be given. The participants were either PrGs in treatment for gambling problem, current HSs or HCs. Importantly, PrGs had to be at least one week abstinent from gambling, and HSs had to be overnight smoking abstinent (16-18 hours of abtinentce). Participants filled out the gambling and smoking urge questionnaires before and immediately after fMRI scanning.

This study highlighted that viewing gambling pictures (as opposed to neutral pictures) is related to brain activation in left occipital cortex, bilateral parahippocampal gyrus, right amygdala and right in PrGs relative to HSs and HCs. Importantly, within the PrG group, a positive relationship was found between gambling-related craving (only post-scan scores were used) and activation in the VLPFC, left anterior insula and left caudate head when viewing gambling pictures, as compared to neutral pictures. In addition, Goudriaan et al. [44] observed increased brain activity to smoking cues (VMPFC, rostral ACC and left VLPFC) in HSs with high levels of nicotine dependence, as compared to HSs with lower levels of nicotine dependence (left precuneus, right insula, left middle and superior temporal gyri) and with PrGs and HCs (VMPFC, rostral ACC and left VLPFC). Moreover, higher smoking urge in HSs was associated with increased activity in the VLPFC and left amygdala during viewing of smoking-related pictures versus neutral pictures. Nevertheless, no significant difference was observed between pre-scan and post-scan scores of gambling or smoking urge in PrGs and HSs, respectively.

- 2012: Balodis et al. [42]. This study aimed to examine the association between emotion and motivational ratings and neural cue reactivity. The data was the same as in Potenza et al. [40]. Indeed, another interesting aspect from the task design in Potenza et al. [40] is that participants were asked to rate the intensity of their emotional or motivational responses triggered by the video scenarios of sadness, happiness or gambling. For each scenario, participants were instructed to push a button when they started to feel sadness, happiness or an urge to gamble, respectively. Then, following each video, participants described the quality of their emotional or gambling urge responses and rated them on a 10-points Likert scale. In Potenza et al. [40], subjective reports and brain imaging data were analyzed separately, with the PrG group reporting stronger emotional responses and gambling urges when viewing the gambling scenarios, as compared to the HC group. Therefore, Balodis et al. [42] extended the findings from 
Potenza et al. [40], by examining the association between brain imaging data (according to the three scenario types and to the three epochs of interest) and participants' subjective ratings of the video scenarios.

Balodis et al. [42] observed that correlations between self-reported responses and brain activations were strongest during the epoch corresponding to the middle viewing period, and more robust in PrGs than in HCs for all conditions. During this epoch, subjective ratings of gambling urges in the PrG group were negatively correlated with MPFC activation and positively correlated with middle temporal gyrus and temporal pole activations. Sadness ratings in the PrG group correlated positively with activation of the medial orbitofrontal cortex, middle temporal gyrus, and retrosplenial cortex, while self-reported happiness during the happy videos demonstrated mainly inverse correlations with activations in the temporal poles. However, although this study employed a significance threshold of 0.7 for the correlational analyses altogether with an extent threshold of 25 contiguous voxels for selecting clusters of activation, Type I errors remain an issue due to the high numbers of correlations undertaken (not reported). The use of a single rating of subjective urges to gamble is another caveat, as it does not disentangle levels of gambling urge felt prior and after the viewing of the gambling scenario.

- 2012: Balodis et al. [45]. This study used a "monetary incentive delay task" (adapted from [45]), which consisted of (i) a reward prospect, (ii) a motor-action, (iii) an anticipation phase, and (iv) an outcome phase. In the reward prospect phase, participants (a group of PrGs and a group of HCs) viewed a cue signaling the potential to win or lose money. In the motor-action phase, participants had to simply press a button when a target appeared. Participants won (or avoided losing) money by pressing a button before the target disappeared. In the anticipation phase, participants waited for feedback notifying whether they had won or lost the trial. In the outcome phase, participants received feedback on whether they had won or lost the trial as well as on their cumulative earnings. Task difficulty (i.e., the length of target presentation during the motor-action phase) was based on reaction times collected during a pre-scan practice session, such that participants won on $66 \%$ of trials.

During the reward prospect phase (i.e., signaling a potential win or loss), between-groups contrast revealed decreased brain activity in PrGs relative to HCs in the medial PFC, the VMPFC, the insula, the ACC, the left VS, and in the left inferior frontal gyrus. During the anticipation phase (win condition only), PrGs exhibited decreased brain activity in the left VMPFC extending to the VS. PrGs also demonstrated decreased activations in multiple regions of when receiving a monetary win or loss during the outcome phase.

- 2012: Van Holst et al. [48]. This study used a "guessing task" (adapted from [56]), which consisted of three phases: (i) expectation, (ii) anticipation, and (iii) outcome. In the expectation phase, participants (a group of treatment-seeking PrGs and a group of HCs) viewed a cue signaling a probability to receive a monetary reward and had to indicate with a button press whether they expected to win or lose. Then, participants had to wait 4 seconds (i.e., the anticipation phase) to receive a win or loss feedback (i.e., the gambling outcome phase).

In the brain imaging analyses, van Holst et al. [40] merged the expectation and anticipation phase (total epochs length $=6$ seconds). Betweengroup analyses revealed that, as compared with $\mathrm{HCs}$, PrGs exhibited increased activations in the bilateral DS and the left orbitofrontal cortex (OFC) when they expected and anticipated a monetary gain. Importantly, within the PrG group, gambling problem severity was negatively associated right amygdala when expecting and anticipating a monetary gain. van Holst et al. [53] did not report the findings regarding brain activations in the outcome phase.

- 2012: van Holst et al. [46]. This study examined the interaction between gambling cue reactivity and motor response inhibition in PrG. Speфifically, a group of treatment-seeking PrGs and a group of HCs performed a Go/No-go task that required to press a button when a certain type of stimulus (neutral, positive, negative or gambling pictures) was shown (Go trials) and to inhibit pressing the button when a neutral stimulus appeared (No-Go trials). Hence, the Go/No-go task consisted of four blocks containing pictures that were positive, negative, neutral, or gamblingrelated. Because all pictures were neutral in the neutral block, participants were instructed to respond to all neutral pictures, but not to respond when a vehicle was shown in the picture.

van Holst et al. [46] observed higher DLPFC, ACC and VS activations for the gambling cues (i.e., go trials from the gambling block) minus control cues (i.e., go trials from the neutral block) contrast in PrGs, as compared with HCs. Importantly, PrGs were also better than HCs at inhibiting their motor response in blocks featuring neutral pictures (i.e., no/go trials) and gambling pictures (i.e., go trials). Moreover, as compared with HCs, PrGs showed lower activation of the DLPFC and ACC regions during no-go trials (i.e., neutral pictures) of the gambling blocks than during no-go trials of the neutral blocks. One explanation for this result is that this sample of gamblers was recruited from addiction treatment centers, where they received cognitive behavioral therapy. This could have lowered their motivational-approach tendencies when embedded into a gambling context (see also [57]).

- 2012: van Holst et al. [47]. This functional connectivity study relied on the dataset from van Holst et al. [46]. Group interactions showed that during neutral inhibition, HCs exhibited greater functional connectivity between the left caudate and occipital cortex compared with PrGs. In contrast, during inhibition in the positive condition, PrGs showed greater functional connectivity between the left caudate and occipital cortex compared with HCs. During inhibition trials in the negative condition, a stronger functional connectivity between the left caudate and the right ACC in PrGs relative to HCs was present.

- 2013: Sescousse et al. [49]. A group of PrGs and a group of HCs participated to an "incentive delay task" [101]. Each trial consisted of an (i) anticipation, a (ii) discrimination, and (iii) an outcome phase. During the anticipation phase, participants saw a cue announcing the type (either monetary or erotic), probability (0/25/50/75\%) and intensity (low/high) of an upcoming reward. In the next phase, participants were asked to perform a visual discrimination task (left button press for a triangle; right button press for a square) within a maximum time of 1 second. Success on this task preserved the participants' chance to obtain the probabilistic reward. In the outcome phase of the rewarded trials, participants saw an erotic image (with high or low erotic content) or a cue mentioning the amount of money won (high or low amount). In addition, following each reward outcome, participants were asked to provide a hedonic rating on a $1-9$ continuous scale $(1=$ very little pleased; $9=$ very highly pleased $)$. In non-rewarded and control trials, participants were presented with "scrambled" pictures.

In the anticipation phase, the monetary versus erotic cues contrast revealed an increased response in PrGs relative to HCs in the VS, which appeared largely driven by a reduced sensitivity to erotic cues. Moreover, within the PrG group, the intensity of the differential response to monetary versus erotic cues in the VS was associated with problem gambling severity.

In the outcome phase, between-group analyses highlighted increased OFC activation in PrGs when receiving a monetary gain. Sescousse et al [49] also examined the modulation of brain activation by the hedonic ratings. For monetary rewards, they found that activity in the VS correlated with hedonic ratings in both $\mathrm{HC}$ and PrG participants. By contrast, VS activation in HCs, but not in PrGs, varied according to the hedonic ratings of erotic rewards. This finding suggests that the VS of PrGs failed at encoding the hedonic value of erotic rewards.

- 2016: Kober et al. [50]. This study followed prior work from Potenza et al. [40] and Wexler et al. [54] with participants viewing videos depicting cocaine, gambling, or sad scenarios. As compared to previous work from this research group, this study examined neural cue reactivity in larger 
in larger samples of CDs, PrGs and HCs. Participants viewed six videos depicting cocaine, gambling, and sad scenarios presented in a counterbalanced order. After each video, participants were asked to rate their urge to use cocaine or to gamble on a $1-10$ scale $(1=$ not at all, $10=\mathrm{a}$ lot $)$. Each functional run was divided into two periods of video viewing: the initial 45 seconds of video viewing (to examine the emergence of craving/emotional responses), and the final 45 seconds of video viewing (to examine more developed or protracted craving/emotional responses).

Between-group analyses related to in-scanner subjective ratings revealed that CDs reported highest cocaine urges in response to cocaine videos and PrGs reported highest gambling urges in response to gambling videos. Neuroimaging data revealed that the initial period of video viewing activated the ACC and VMPFC, and predominantly to cocaine videos in CDs. In the last period of video viewing the DLPFC and the dorsal ACC were most strongly activated for cocaine videos in CDs, gambling videos in PrGs, and for sad videos in HCs. No correlation analyses were reported between craving scores and brain imaging data.

- 2017: Limbrick-Oldfield [47]. In this study, a group of PrGs in treatment and a HC group were scanned while viewing gambling, gamblingmatched neutral, food, or food-matched neutral pictures. There were four subtypes of gambling cues: photographs of the shop-fronts of bookmakers, as well as 'action' images from electronic roulette, sports betting and slot machines. For each PrG participant, Limbrick-Oldfield et al. [47] selected the two forms most relevant to PrGs' personal game preferences, as well as the shopfronts. To control for the potential impact of fasting on neural responses to food and gambling cues, participants were instructed to eat a light meal $\sim 2 \mathrm{~h}$ before the scan.

Stimuli were presented in a blocked design. Each block contained five images from the same category. Participants were instructed to imagine that they were in the place pictured in each photograph or interacting with the item shown. Moreover, to maintain attention, participants were asked to press a button with each new image. At the end of each block, participants gave a craving rating ("I crave gambling right now") on a 1-9 scale $(1=$ strongly disagree, $9=$ strongly agree $)$. They were also asked to rate their craving to gamble before they entered the scanner.

Within the PrG group, brain imaging analyses on the contrast of gambling minus gambling-matched neutral cues revealed increased activity within the left posterior cingulate gyrus, the left superior frontal gyrus, the left frontal pole and extended to multiple regions including the bilateral VS, MPFC, left angular gyrus and right lateral occipital cortex. For the same contrast, and compared with $\mathrm{HCs}$, PrGs showed increased activity in the left insula, the left frontal operculum, ACC and superior frontal gyrus.

Limbrick-Oldfield et al. [47] also undertook brain connectivity analyses between the nucleus accumbens and ROI including the PFC, insula and VS. Within PrGs, the contrast of gambling minus gambling-matched neutral cues revealed increased functional connectivity between the nucleus accumbens and the right inferior frontal gyrus. Between-group analyses showed increased functional connectivity, compared with HCs, between the nucleus accumbens and the left insula cortex (extending to left putamen), and the superior frontal gyrus.

PrGs also exhibited higher mean craving scores than HCs after the viewing of gambling-related pictures. PrGs also showed a significant craving increase following gambling cues relative to both neutral cues and rest blocks. At the brain level, for the gambling minus gamblingmatched neutral cues contrast, mean craving ratings in the PrG group were associated with greater activity within the right insula, the left central operculum/left insula, the cerebellum, and the ROI mask in the nucleus accumbens. For the functional connectivity analysis, higher craving ratings were associated with reduced connectivity between nucleus accumbens and medial PFC. No region showed a significant correlation with problem gambling severity.

- 2017: Brevers et al. [48]. This study examined whether the viewing of gambling-related pictures impacts on proactive (the restrain of actions in preparation for stopping) and reactive (outright stopping) inhibition. A group of high-frequency poker players, and one group of matched nongambler controls, performed a modified version of the stop-signal paradigm, which required participants to inhibit categorization of poker or neutral pictures. The probability that a stop-signal occurs $(0 \%, 17 \%, 25 \%, 33 \%)$ was manipulated across blocks of trials, as indicated by the color of the computer screen.

Behavioral analyses revealed that poker players were faster than controls in categorizing pictures across all levels of proactive motor response inhibition (go trials). Brain imaging analyses highlighted higher dorsal ACC activation in poker players, as compared with controls, during reactive inhibition. Taken together, findings from Brevers et al. [48] suggest that, due to their faster rates of stimulus discrimination (i.e., go responses), poker players might have recruited more cognitive resources than controls when required to stop their response (reactive inhibition). In other words, these findings suggest that frequent gamblers need to trigger additional cognitive resources, when required to stop their motor response, while being embedded in an environment featuring gambling stimuli. Nevertheless, Brevers et al. [48] did not observe any significant effect of stimulus type (control vs. poker-related), at both behavioral and neural levels. This suggests that the observed effects were due to a familiarity bias (e.g., high expertise in discriminating poker cues) rather than to salient-motivational processes.

Notes. $C D$ cocaine dependent; $H C$ healthy control; $H S$ heavy smoker; $\operatorname{Pr} G$ problem gambler; $R O I$ regions of interest; $A C C$ anterior cingulate cortex; $D L P F C$ dorsolateral prefrontal cortex; $D S$ dorsal striatum; $M P F C$ medial prefrontal cortex; $O F C$ orbitofrontal cortex; $P F C$ prefrontal cortex; $V M P F C$ ventromedial prefrontal cortex; $V L P F C$ ventrolateral prefrontal cortex; $V S$ ventral striatum; $V L T$ video lottery terminals

craving elicited by the immediate availability of cigarettes was diminished by transiently reducing DLPFC activity through transcranial magnetic stimulation. This further suggests that brain regions commonly associated with reflective processes do not only support inhibitory control, but more generally modulate mesolimbic value signals up or down based on goals and context.

In the past decade, research has shown that gamblingrelated cues can foster the development of strong attentional biases and positive memory associations among problem gamblers (for a review, see [37-39]). The pivotal role of incentive salience-related processes in gambling disorder is further evidenced by the fMRI literature on gambling cue reactivity, covered in the next section.

\section{Gambling Cue Reactivity Paradigms in Neuroimaging Research}

Table 1 and Box 1 offer a comprehensive account of the experimental paradigms used in fMRI studies to examine gambling cue reactivity. Despite the limited literature in comparison with the one available for substance use disorder (e.g., [53]), a highdiversity of experimental designs been used to examine the neural correlates of gambling cue reactivity. These studies have been undertaken in sub-clinical individuals as well as individuals with severe gambling disorder (collectively referred to here as problem gamblers; PrGs), being either active or abstinent PrGs at testing time, and usually compared with groups of non-gambler healthy controls (HCs; excepted in $[40,50]$ where 
a group of PrGs was compared with a group of individuals with cocaine addiction and a group of healthy controls).

Similarly to what has been done in the field of substance use disorder (e.g., [9, 12]), a key feature of cue reactivity paradigms is that they expose participants to gambling-related cues, depicting real-life gambling-related situations through auditory and/or visual stimulations [40, 41, 43, 44, 46, 50-52]. These gambling cues are matched either to control cues [41, 43, 44, $46,51,52]$ or to other types of motivationally-salient (e.g., food, smoking) or emotionally-laden (e.g., happy, sad) cues [40, 41, $44,46,49,50,52 \bullet \bullet$. In some studies, gambling pictures were customized according to participants' gambling preferences [51, 52••]. Gambling-related pictures were used either as taskirrelevant (i.e., distracting) [44] or as task-related targets during stimulus discrimination task [46]. Several studies instructed participants to picture themselves experiencing the gambling situation $[40,41,50,52 \cdot \bullet]$ or to passively watch the gambling cues while being informed that they will have the opportunity to gamble after the scanning session [43]. As outlined in Box 1, this high-diversity of methodological approaches likely plays a role in the inter-studies variability of the activation maps reported.

Another important aspect of neural cue reactivity studies is that brain activation patterns were generally correlated with subjective self-reports. In the fMRI literature on gambling cue reactivity, these ratings are supposed to measure gamblingrelated craving [43, 44, 50, 52••], and/or emotional and motivational responses $[40-42,50]$. This type of measure is usually collected after each task run/block [40-42, 50], before the scanning session and after each task block/run [43, 52••], or pre- and post-scanning session [44].

In addition to studies using classical cue reactivity tasks, fMRI was also used to examine the neural correlates of motor response inhibition toward gamblingrelated cues [46, 47, 51]. This type of studies allowed to identify how effortful and cognitive control processes impact upon neural gambling cue reactivity. Lastly, brain imaging studies also involved cues signaling the occurrence of probabilistic monetary rewards, allowing to probe anticipation-related brain activity [45, 48, 49].

\section{fMRI Evidence of Gambling Cues Reactivity}

Findings summarized in Box 1 outline that exposure to (audio)visual gambling cues elicit increased brain activations in individuals with problem gambling relative to non-gambler matched controls. Only Potenza et al. [40] (see also [41, 42] using the same dataset) and Balodis et al. [45] observed diminished neural gambling cue reactivity in PrGs relative to HCs.

One potential explanation for this discrepancy is that, in Potenza et al. [40], both within-subject and between-group contrast images were computed using uncorrected thresholds across the whole brain. Hence, while Potenza et al. [40] provided preliminary evidence of gambling cue reactivity, it cannot be excluded that type I errors occurred due to the relatively lenient statistical threshold used (e.g., [59]). Interestingly, using a similar task design as in Potenza et al. [40], Kober et al. [50] observed opposite results, i.e., increased activation in the dorsomedial prefrontal cortex and dorsal anterior cingulate cortex of PrGs while exposed to gamblingrelated videos. The larger sample size and the whole-braincorrected thresholds used in this latter study make the results potentially more reliable. Balodis et al. [45] observed that PrGs exhibit decreased striatal activations when viewing cues indicating potential monetary gains or losses. These findings differ from those obtained by van Holst et al. [48] and Sescousse et al. [49], which highlighted increased patterns of striatal activations when PrGs were exposed to such cues. Nevertheless, the experimental tasks used in these fMRI studies differed according to the level of uncertainty associated with monetary outcomes. Specifically, in van Holst et al. [48] and Sescousse et al. [49], the monetary reward was probabilistic (i.e., explicit win/loss ratio followed by a random draw), which is comparable to a real-life gambling (e.g., [60]). By contrast, in Balodis et al. [45], participants viewed a cue signaling the potential to win or lose money, which was entirely contingent on a button press (i.e., participants had control over the reward delivery process). As such, the decreased pattern of brain activation observed in PrGs by Balodis et al. [45] might be due to less realistic and ecological gambling scenarios (for additional discussions, see [61-63]). This suggests that PrGs attribute high incentive salience toward cues that are intimately related to gambling, but show decreased interest toward cues signaling the availability of a conventional monetary reinforcement. In other words, the processes of incentive salience attribution may be restricted to a narrow set of cues intimately related to gambling (e.g., [64, 65]). However, one should note that this reasoning is based on a reverse inference and should thus be taken with caution.

A central finding of past fMRI studies is the consistent association, within the PrG group, between cueinduced brain activations, disordered gambling symptoms [49], and gambling craving (task-induced craving change [52••]; post-task craving scores [44]; gambling craving rating scores obtained after the viewing of gambling video [42]). These findings are of critical importance as they suggest that brain reactivity to gambling cues is a valid biomarker of gambling-related craving and of gambling disorder severity. Noteworthy, Kober et al. [50] and Crockford et al. [43] did not report analyses on the association between brain activation and gambling craving ratings, despite having reported group differences regarding craving scores in PrGs relative to $\mathrm{HCs}$.

Available fMRI literature on gambling cue reactivity also shows that integrating different types of hedonic cues, within 
the same task design, impacts on the magnitude of the main and interactive effects of brain imaging results [44, 46, 49, $52 \bullet \bullet$. For instance, findings from Sescousse et al. [49] suggest that the concurrent availability of monetary and erotic rewards triggered a motivational hierarchy favoring monetary rewards over erotic ones in PrGs. Similar findings were found in a study comparing patients with gambling disorder or substance use disorder with regard to gambling versus cocaine cue reactivity [50]. Specifically, this study showed that the dorsomedial prefrontal cortex and the dorsal anterior cingulate cortex were most strongly activated for cocaine-related videos in cocaine-dependent participants, and for gambling videos in PrGs, which clearly suggests a specificity of brain reactivity to the cues associated with the addictive behavior.

As a whole, given the robust evidence that brain activity in PrGs is strongly modulated by gambling cues, we believe that the examination of the neural reactivity toward gambling cues represents a promising tool for clinical neuroscience of gambling disorder.

\section{An Integrative Framework for Examining Neural Cue Reactivity in the Age of Online Gambling}

In comparison to the literature on neural cue reactivity in substance use disorder, available knowledge on the key factors underlying cue reactivity in gambling disorder is still very incomplete. Specifically, it is currently unclear how individual-specific factors (symptom severity, duration/ intensity of use, active user vs. trying to quit, treatment outcomes) and study-specific factors (e.g., craving induction, reward availability, personalized cues) impact on gambling cue reactivity. Therefore, our aim here is to provide direct research directions for enhancing current knowledge on how specific factors impact on gambling cue reactivity, and by extension on its predictive power regarding clinical status and treatment outcome of gambling disorder.

Capitalizing on influential model-based reviews on neural cue reactivity in substance use disorder $[9,11,12,66]$, the following sections describe a conceptual and methodological framework that attempts to integrate both individual-specific and study-specific factors known to modulate neural cue reactivity in cocaine, alcohol, and nicotine users (see also Table 2 for a summary of the proposed research directions). While implementing this integrative approach in experimental research presents important challenges, we argue that the recent expansion and popularization of online sports betting services calls for the development of more comprehensive and specific models of neural cue reactivity in gambling disorder.

\section{Exploring the Clinical Validity of Gambling Cue Reactivity}

There is currently a rapid proliferation of sports betting opportunities. One striking feature of this new offer of online gambling is the advent of in-play betting that allows sports bettors to place bets during the game (e.g., on the final outcome of the game, on key events within the game, or on a particular discrete event during a game; [67]). As such, a countless number of sport events continuously promote gambling opportunity, a phenomenon that has already been linked to an increased willingness to bet in sport fans (including children; [8•, 68-71]). Moreover, in contrast to other types of gambling activities, sports betting is not negatively connoted in our society (e.g., [72]). Hence, both the hyper-accessibility and the increase level of social acceptance of this conduct can be expected to expand the spectrum of gamblers within the population, with specific samples of gamblers (i.e., sport bettors) at both extreme ends of the spectrum of gambling frequency and severity.

Table 2 Summary of the proposed integrative framework for examining neural cue reactivity in the age of online gambling

\begin{tabular}{|c|c|}
\hline Identified challenges & Potential research directions \\
\hline $\begin{array}{l}\text { Exploring the clinical validity } \\
\text { of gambling cue reactivity }\end{array}$ & $\begin{aligned}=> & \text { Identify how neural cue reactivity varies as a function of the duration/intensity of gambling behavior, } \\
& \text { the degree of gambling disorder severity, and the current status (active user, trying to quit, abstinent) }\end{aligned}$ \\
\hline $\begin{array}{l}\text { Establishing the predictive value } \\
\text { of gambling cue reactivity }\end{array}$ & $\begin{aligned}= & \text { Examine whether neural reactivity to gambling cues, measured before an attempt to quit, could identify } \\
& \text { problem gamblers with heightened relapse vulnerability } \\
= & >\text { Examine whether neural reactivity to gambling cues, measured before the actual initiation of gambling habits, } \\
& \text { could predict later problematic gambling behaviors }\end{aligned}$ \\
\hline $\begin{array}{l}\text { Integrating new measures } \\
\text { of gambling involvement }\end{array}$ & $\begin{aligned}=> & \text { Examine how neural reactivity to gambling cues varies as a function of harmonious versus obsessive } \\
& \text { passion for gambling }\end{aligned}$ \\
\hline $\begin{array}{l}\text { Using cues associated with } \\
\text { gambling availability }\end{array}$ & $\begin{aligned}= & >\text { Examine how the prospect of actual gambling impacts on neural reactivity to gambling cues } \\
=> & \text { Examine the interactions between cue-elicited feelings (e.g., enjoyment, winning confidence) } \\
& \text { and pre- versus post-task changes in gambling craving }\end{aligned}$ \\
\hline $\begin{array}{l}\text { Renewing measures } \\
\text { of key variables }\end{array}$ & $\begin{aligned}=> & \text { Examine whether gambling cues associated with ubiquitous touchscreen smartphone apps impact } \\
& \text { on neural cue reactivity during motor response inhibition }\end{aligned}$ \\
\hline
\end{tabular}


From the standpoint of the present review, this variation in gambling participation and severity calls for a new line of research for further establishing the clinical validity of gambling cue reactivity with indices of duration/intensity of use, addiction severity, and current clinical status (active user, trying to quit, abstinent). All these individual-specific factors are known to modulate neural reactivity to psychoactive substance cues in substance use disorder. For instance, while reviewing fMRI studies of drug cue reactivity, Wilson et al. concluded that drugrelated cues trigger increased brain activation in individuals who are actively using drugs and not seeking treatment at testing time, as compared with treatment-seeking drug users [11]. Moreover, several studies observed that levels of hedonic/ incentive cognitive association [73] and motor approach tendency toward alcohol cues [74-78] are lower in individuals who are motivated to quit, as compared with heavy alcohol users. These patterns can be accounted for if one assumes that quittingmotivated individuals, in contrast to active users, develop an active avoidance strategy toward cues to support their abstinence/moderation goals [76]. Taken together, these experimental approaches contrast with fMRI studies on gambling cue reactivity, which have often compared one sample of PrGs (either active or treatment-seeking) with a group of non-gambler HCs, eventually failing to identify brain pathways that vary according to frequent (but non-problematic) and problematic gambling habits.

\section{Establishing the Predictive Value of Gambling Cue Reactivity}

One main challenge for future research is to establish whether neural reactivity to gambling cues (not only related to sports betting but also to other gambling types), measured before an attempt to quit, could identify gamblers with heightened relapse vulnerability. Previous research on substance use disorder have already shown that relapse-vulnerable individuals can be identified before quit attempts based on their brain reactivity to substance-related cues (for a review, see [9]). For instance, Janes et al. [79] highlighted that the insular cortex response to smoking cues before trying to quit was a significant predictor of relapse in quitting-motivated smokers.

This line of research should not only focus on treatment outcomes, but also on examining whether neural cue reactivity to gambling cues predicts problematic gambling behaviors. This type of studies appears especially relevant to the field of sports betting. Specifically, recent research findings have highlighted that despite having never gambled, some young sports fans displayed technical knowledge of sports betting, including being able to discuss and describe "odds," different gambling markets, and how to place bets [69]. This betting-related knowledge could be predominantly traced back to the abiding marketing they were faced with (e.g., pop-up messages occurring during live sports events that feature dynamic betting ratios; [80]), inducing increased recall and awareness of sports betting brands, or perceptions of promotional strategies $[68,69,81,82]$. As such, this ubiquity of cues might increase the incentive salience of sports betting in young individuals long before they reach the minimum legal age for gambling. In this context, neuroimaging research could prove useful to examine whether neural cue reactivity at time 1 (e.g., in young people who are sport fans but are not legally authorized to bet) predicts the problematic involvement in sports betting at time 2 (e.g., 1 year after being legally authorized to bet on sports). Ultimately, this type of research should enable the creation of personalized prevention and treatment programs on problematic sports betting.

\section{Integrating New Measures of Gambling Involvement}

Brain imaging studies on gambling cue reactivity will also benefit from alternative measures of gambling habits. One important aspect is to characterize samples of gamblers according to their actual involvement in gambling, as "high involvement" is not necessarily associated with negative consequences or disordered gambling symptoms (e.g., [83]). Indeed, past research has shown that it is possible to distinguish harmonious passion (i.e., a strong inclination to engage in the activity willingly and with a sense of volition) from obsessive passion (i.e., an uncontrollable urge to engage in the activity; [84-86]) toward sports (e.g., with the "Sports Fans Passion Scale"; [87]) and sports betting (e.g., with a sports betting adaptation of the "Gambling Passion Scale"; e.g., [88]). Considering this critical difference between harmonious and obsessive passion is of major importance when examining cue reactivity processes in individuals who aim at controlling or stopping sports betting. Specifically, one key aspect of sports betting is that it binds gambling to watching sport, that is, a popular, enjoyable, and valorized activity. Hence, a challenge for these quitting-motivated sports bettors is to restore an interest in sports events watching per se, that is, without betting on it. This view echoes qualitative studies that examined trajectories of recovery from gambling problems [89-91, 92•]. Specifically, these studies highlighted that processes of behavioral change revolve around shifting from a "gambling self" to a self-identity that is reshaped in harmonious and appropriate ways. In terms of brain-related clinical outcome, one would expect such a shift to be accompanied by diminished brain reactivity to sports betting cues combined with increased brain activity toward sports watching cues in abstinent sports bettors, as compared with active problem sports bettors.

\section{Using Cues Associated with Gambling Availability}

Since every sporting event is available to bet on, merely viewing cues related to sporting events (e.g., advertisements featuring betting odds) has the potential to drastically increase 
gambling temptation [5, 6, 93-95]. In other words, exposure to sports betting cues signals gambling availability.

Research is thus warranted to extend previous neuroimaging work on gambling cue reactivity by examining how the prospect of actual betting impacts specific brain pathways. Initial strides toward this research direction have been taken by Brevers and colleagues [96••]. These authors reported, through the use of an fMRI cue exposure task (adapted from a food cue reactivity study; [97]), that thinking about a sporting event with the intention of gambling on the outcome, compared with thinking about it with the mere intention of watching it, triggers higher prefrontal, insular, and striatal activations in a sample of football (soccer) fans. Importantly, Brevers et al. [96••] used ecological cues (i.e., football games that were occurring in real life in the days following the scan, with the logos and names of the two teams facing each other) and manipulated gambling reward availability/expectancy (participants received additional money if the team they chose to bet on eventually won the game). Comparable study-specific factors (e.g., substance availability/expectancy; substance cues as task target; personalized cues) have been shown to modulate neural cue reactivity in substance use disorder (e.g., [9]).

Another interesting feature of the Brevers et al. [96••] study is the inclusion of post-task ratings for individual cues that were regressed against brain responses observed during the experiment. Two ratings were used: the degree of confidence toward the winning team and the degree of enjoyment directed toward a game. Indeed, all sports fan can express a degree of confidence toward the result of a forthcoming sport event (e.g., through "Fantasy Sports Leagues" with or without monetary/material reward involved; [98]). We advance that similar procedures should be used in future studies to complement pre- and post-task (block) craving measures. This would allow to take into account the interaction between the level of interest elicited by the cues and pre- versus post-task craving changes. In addition, including such parametric indices would represent a considerable advantage for experimental tasks that alternate reward availability conditions on a trial-per-trial basis, including exposure to situations known to interact with neural cue reactivity as a potent trigger of impulsive gambling behaviors (e.g., reward-blocking or frustration induction procedures; [98-101]). For instance, individuals are more impulsive in their monetary choices after having experienced "frustration", e.g., when they are denied a gambling opportunity [100]. Accordingly, sports bettors should experience similar heightened frustration when they perceive a cue depicting an attractive yet unavailable betting opportunity. Previous neuroimaging studies have shown that the insular cortex and the amygdala play a key role in evaluating the emotional content triggered by these "frustrating" events [101, 102]. As such, this new line of research may extend current knowledge on the brain pathways underlying situations that fuel gambling temptation.
Renewing Measures of Previously Explored Variables

Another central aspect of the new sports betting offer is that recent technological advances allow for repeated and continuous access to sports betting at the touch of a smartphone screen (i.e., mobile gambling; [79]). As such, the motor response pattern used for opening a sports betting smartphone apps mimics the button press procedures commonly used in the laboratory (e.g., to push the left or the right computer key; go or no-go responses). This opens new avenues for ecological behavioral and brain imaging research examining the interaction between cue reactivity and motor response inhibition in the lab. For instance, the go/no -go task used by van Holst et al. [46] or the stop-signal paradigm chosen by Brevers et al. [51] can easily be adapted with cues depicting print screens from sports betting apps taken from participant's smartphones (with print screens from other apps to be used as control cues; e.g., mail, calendar, notes). Indeed, it has already been shown that cues associated with ubiquitous touchscreen smartphone apps trigger heightened sensorimotor skills and strong motor approach tendencies (e.g., $[103,104])$. It follows that the extensive use of online sports betting platforms could impair the ability to stop a motor response when it interferes with updated goal-driven behaviors (e.g., to withdraw or refrain a motor response toward a sports betting cue).

\section{Adopting a Data Driven Approach in the Age of Open Science}

Capitalizing on sports betting cues will enhance the validity of cue reactivity tasks. Nevertheless, it is important to methodological considerations while using a stepwise approach (e.g., running behavioral pre-tests and pilot neuroimaging studies before undertaking neuroimaging studies) and integrated levels of data analyses (from whole-brain and functional connectivity analyses to ROI and effective connectivity analyses, respectively). For instance, brain Z-maps from Brevers et al. [96••] could be used as functional masks by future studies when assessing group activation differences in predefined regions of interest. This should be especially helpful for increasing the statistical power of future studies involving participants with high levels of problematic sports betting habits - that is, those who are difficult to recruit, usually resulting in small and underpowered samples. A comparable approach has been adopted in brain imaging research on gambling disorder by Sescousse et al. [49], who used peak voxels from their previous study using the same protocol in healthy controls [58] to draw ROI spheres. This procedure allowed them to identify interactions among the brain networks involved in the processing of salient-motivational cues in PrGs. Another promising avenue is the creation of multicenter brain research projects (e.g., [105•]) and neuroscience-based framework for gambling disorder (such as those already existing in 
substance use disorder; $[106,107])$. These initiatives can now be more easily implemented by using pilot data for computing the necessary sample size to obtain a certain level of statistical power (e.g., through the use of http://neuropowertools.org and http:// fmripower.org) prior to submitting preregistered reports and by sharing research materials online, with the statistical maps that can be uploaded to neurovault.org, the raw data to openfmri.org, and the code to github.com.

\section{Concluding Remarks}

Experimental designs investigating individual-specific and study-specific factors related to sports betting have the potential to offer a fine-grained approach to the examination of neural gambling cue reactivity. We are convinced that this integrative approach will not only increase our understanding of the neurobiology of problem gambling severity, treatment outcome, and relapse risk in gambling disorder, but will also help in identifying biomarkers that can disentangle between harmonious and harmful gambling habits. Ultimately, along with inputs from open science initiatives building upon multicenter collaborations, this scientific work should speed up the implementation of efficient public health prevention and treatment programs on new forms of gambling disorder.

Acknowledgments This work was supported by the Luxembourg National Research Fund (FNR); CORE - Junior Track [BETHAB].

\section{Compliance with Ethics Guidelines}

Conflict of Interest Damien Brevers, Guillaume Sescousse, Pierre Maurage, and Joel Billieux declare no conflicts of interest relevant to this manuscript.

Human and Animal Rights and Informed Consent This article does not contain any studies with human or animal subjects performed by any of the authors.

\section{References}

Papers of particular interest, published recently, have been highlighted as:

- Of importance

• Of major importance

1. Armstrong AR, Thomas A, Abbott M, Gambling Participation. Expenditure and risk of harm in Australia, 1997-1998 and 20102011. J Gambl Stud. 2018;34(1):255-74.

2. Pitt H, Thomas SL, Bestman A. Initiation, influence, and impact: adolescents and parents discuss the marketing of gambling products during Australian sporting matches. BMC Public Health. 2016;13(16):967.
3. Shaffer HJ. From disabling to enabling the public interest: natural transitions from gambling exposure to adaptation and self-regulation. Addiction. 2005;100(9):1227-30.

4. Shaffer HJ, LaPlante DA, LaBrie RA, Kidman RC, Donato AN, Stanton MV. Toward a syndrome model of addiction: multiple expressions, common etiology. Harv Rev Psychiatry. 2004;12(6):367-74.

5. Hing N, Russell AM, Browne M. Risk factors for gambling problems on online electronic gaming machines, race betting and sports betting. Front Psychol. 2017;8:779.

6. Hing N, Russell AMT, Lamont M, Vitartas P. Bet anywhere, anytime: an analysis of internet sports bettors' responses to gambling promotions during sports broadcasts by problem gambling severity. J Gambl Stud. 2017;33(4):1051-65.

7. Columb D, O'Gara C. A national survey of online gambling behaviours. Irish J Psychol Med. 2017;351-9.

8. Sharman S, Murphy R, Turner JJD, Roberts A. Trends and patterns in UK treatment seeking gamblers: 2000-2015. Addict Behav. 2019;89:51-6 This study offers compelling evidence that the mental health and gambling habits of problem gamblers have changed in the last fifteen years, sports betting and poker being increasingly reported as problematic.

9. Jasinska AJ, Stein EA, Kaiser J, Naumer MJ, Yalachkov Y. Factors modulating neural reactivity to drug cues in addiction: a survey of human neuroimaging studies. Neurosci Biobehav Rev. 2014;38:1-16.

10. Courtney KE, Schacht JP, Hutchison K, Roche DJO, Ray LA. Neural substrates of cue reactivity: association with treatment outcomes and relapse. Addict Biol. 2016;21(1):3-22.

11. Wilson SJ, Sayette MA, Fiez JA. Prefrontal responses to drug cues: a neurocognitive analysis. Nat Neurosci. 2004;7(3):211-4.

12. Yalachkov Y, Kaiser J, Naumer MJ. Functional neuroimaging studies in addiction: multisensory drug stimuli and neural cue reactivity. Neurosci Biobehav Rev. 2012;36(2):825-35.

13. Strack F, Deutsch R. Reflective and impulsive determinants of social behavior. Personal Soc Psychol Rev. 2004;8(3):220-47.

14. Hofmann W, Friese M, Strack F. Impulse and self-control from a dual-systems perspective. Perspect Psychol Sci. 2009;4(2):162-76.

15. Hofmann W, Gschwendner T, Friese M, Wiers RW, Schmitt M. Working memory capacity and self-regulatory behavior: toward an individual differences perspective on behavior determination by automatic versus controlled processes. J Pers Soc Psychol. 2008;95(4):962-77.

16. Robinson TE, Berridge KC. The neural basis of drug craving: an incentive-sensitization theory of addiction. Brain Res Brain Res Rev. 1993;18(3):247-91.

17. Robinson TE, Berridge KC. Incentive-sensitization and addiction. Addiction. 2001;96(1):103-14.

18. Robinson TE, Berridge KC. Review. The incentive sensitization theory of addiction: some current issues. Philos Trans R Soc Lond Ser B Biol Sci. 2008;363(1507):3137-46.

19. Wiers RW, Bartholow BD, van den Wildenberg E, Thush C, Engels RCME, Sher KJ, et al. Automatic and controlled processes and the development of addictive behaviors in adolescents: a review and a model. Pharmacol Biochem Behav. 2007;86(2):263-83.

20. Wiers RW, Gladwin TE, Rinck M. Should we train alcoholdependent patients to avoid alcohol? Front Psychiatry. 2013;4:33.

21. Berridge KC, Kringelbach ML. Pleasure systems in the brain. Neuron. 2015;86(3):646-64.

22. Hommel B, Wiers RW. Towards a unitary approach to human action control. Trends Cogn Sci. 2017;21(12):940-9.

23. Melnikoff DE, Bargh JA. The mythical number two. Trends Cogn Sci (Regul Ed). 2018;22(4):280-93.

24. Monterosso J, Luo S. Willpower is not synonymous with "executive function”. Behav Brain Sci. 2013;36(6):700-1 discussion 707-726.

25. Bechara A. Decision making, impulse control and loss of willpower to resist drugs: a neurocognitive perspective. Nat Neurosci. 2005;8(11):1458-63. 
26. Droutman V, Bechara A, Read SJ. Roles of the different subregions of the insular cortex in various phases of the decisionmaking process. Front Behav Neurosci. 2015;9:309.

27. Droutman V, Read SJ, Bechara A. Revisiting the role of the insula in addiction. Trends Cogn Sci. 2015;19(7):414-20.

28. Naqvi NH, Bechara A. The hidden island of addiction: the insula. Trends Neurosci. 2009;32(1):56-67.

29. Naqvi NH, Bechara A. The insula and drug addiction: an interoceptive view of pleasure, urges, and decision-making. Brain Struct Funct. 2010;214(5-6):435-50.

30. Naqvi NH, Gaznick N, Tranel D, Bechara A. The insula: a critical neural substrate for craving and drug seeking under conflict and risk. Ann N Y Acad Sci. 2014;1316:53-70.

31. Naqvi NH, Rudrauf D, Damasio H, Bechara A. Damage to the insula disrupts addiction to cigarette smoking. Science. 2007;315(5811):531-4.

32. Noël X, Brevers D, Bechara A. A neurocognitive approach to understanding the neurobiology of addiction. Curr Opin Neurobiol. 2013;23(4):632-8.

33. Noël X, Brevers D, Bechara A. A triadic neurocognitive approach to addiction for clinical interventions. Front Psychiatry. 2013;4:179.

34. Sutherland MT, Carroll AJ, Salmeron BJ, Ross TJ, Hong LE, Stein EA. Down-regulation of amygdala and insula functional circuits by varenicline and nicotine in abstinent cigarette smokers. Biol Psychiatry. 2013;74(7):538-46.

35. Verdejo-García A, Bechara A. A somatic marker theory of addiction. Neuropharmacology. 2009;56(Suppl 1):48-62.

36. Hayashi T, Ko JH, Strafella AP, Dagher A. Dorsolateral prefrontal and orbitofrontal cortex interactions during self-control of cigarette craving. Proc Natl Acad Sci. 2013;110:4422-7.

37. Brevers D, Noël X. Pathological gambling and the loss of willpower: a neurocognitive perspective. Socioaffect Neurosci Psychol. 2013;3:21592.

38. Hønsi A, Mentzoni RA, Molde H, Pallesen S. Attentional bias in problem gambling: a systematic review. J Gambl Stud. 2013;29(3):359-75.

39. Robinson MJF, Fischer AM, Ahuja A, Lesser EN, Maniates H. Roles of "wanting" and "liking" in motivating behavior: gambling, food, and drug addictions. Curr Top Behav Neurosci. 2016;27:105-36.

40. Potenza MN, Steinberg MA, Skudlarski P, Fulbright RK, Lacadie $\mathrm{CM}$, Wilber MK, et al. Gambling urges in pathological gambling: a functional magnetic resonance imaging study. Arch Gen Psychiatry. 2003;60(8):828-36.

41. Potenza MN. Review. The neurobiology of pathological gambling and drug addiction: an overview and new findings. Philos Trans R Soc Lond Ser B Biol Sci. 2008;363(1507):3181-9.

42. Balodis IM, Lacadie CM, Potenza MN. A preliminary study of the neural correlates of the intensities of self-reported gambling urges and emotions in men with pathological gambling. J Gambl Stud. 2012;28(3):493-513.

43. Crockford DN, Goodyear B, Edwards J, Quickfall J, el-Guebaly $\mathrm{N}$. Cue-induced brain activity in pathological gamblers. Biol Psychiatry. 2005;58(10):787-95.

44. Goudriaan AE, de Ruiter MB, van den Brink W, Oosterlaan J, Veltman DJ. Brain activation patterns associated with cue reactivity and craving in abstinent problem gamblers, heavy smokers and healthy controls: an fMRI study. Addict Biol. 2010;15(4):491-503.

45. Balodis IM, Kober H, Worhunsky PD, Stevens MC, Pearlson GD, Potenza MN. Diminished frontostriatal activity during processing of monetary rewards and losses in pathological gambling. Biol Psychiatry. 2012;71(8):749-57.

46. van Holst RJ, van Holstein M, van den Brink W, Veltman DJ, Goudriaan AE. Response inhibition during cue reactivity in problem gamblers: an fMRI study. PLoS One. 2012;7(3):e30909.

47. van Holst RJ, van der Meer JN, McLaren DG, van den Brink W, Veltman DJ, Goudriaan AE. Interactions between affective and cognitive processing systems in problematic gamblers: a functional connectivity study. PLoS One. 2012;7(11):e49923.

48. van Holst RJ, Veltman DJ, Büchel C, van den Brink W, Goudriaan AE. Distorted expectancy coding in problem gambling: is the addictive in the anticipation? Biol Psychiatry. 2012;71(8):741-8.

49. Sescousse G, Barbalat G, Domenech P, Dreher J-C. Imbalance in the sensitivity to different types of rewards in pathological gambling. Brain. 2013;136(8):2527-38.

50. Kober H, Lacadie CM, Wexler BE, Malison RT, Sinha R, Potenza $\mathrm{MN}$. Brain activity during cocaine craving and gambling urges: an fMRI study. Neuropsychopharmacology. 2016;41(2):628-37.

51. Brevers D, He Q, Keller B, Noël X, Bechara A. Neural correlates of proactive and reactive motor response inhibition of gambling stimuli in frequent gamblers. Sci Rep. 2017;7(1):7394.

52.• Limbrick-Oldfield EH, Mick I, Cocks RE, McGonigle J, Sharman SP, Goldstone AP, et al. Neural substrates of cue reactivity and craving in gambling disorder. Transl Psychiatry. 2017;7(1):e992. This fMRI study provides an accurate assessment of gambling cue reactivity, combining personalised gambling cues, the control for the impact of fasting on neural responses to food cues, craving ratings following each task block, whole brain, ROI and functional connectivity analyses, as well as the use of thorough statistical methods for clusterwise inferences.

53. Noori HR, Cosa Linan A, Spanagel R. Largely overlapping neuronal substrates of reactivity to drug, gambling, food and sexual cues: a comprehensive meta-analysis. Eur Neuropsychopharmacol. 2016;26(9):1419-30.

54. Wexler BE, Gottschalk CH, Fulbright RK, Prohovnik I, Lacadie $\mathrm{CM}$, Rounsaville BJ, et al. Functional magnetic resonance imaging of cocaine craving. Am J Psychiatry. 2001;158(1):86-95.

55. Knutson B, Adams CM, Fong GW, Hommer D. Anticipation of increasing monetary reward selectively recruits nucleus accumbens. J Neurosci. 2001;21(16):RC159.

56. Yacubian J, Gläscher J, Schroeder K, Sommer T, Braus DF, Büchel C. Dissociable systems for gain- and loss-related value predictions and errors of prediction in the human brain. $\mathrm{J}$ Neurosci. 2006;26(37):9530-7.

57. Brevers D, Bechara A, Kilts CD, Antoniali V, Bruylant A, Verbanck P, et al. Competing motivations: proactive response inhibition toward addiction-related stimuli in quitting-motivated individuals. J Gambl Stud. 2018;34(3):785-806.

58. Sescousse G, Redouté J, Dreher J-C. The architecture of reward value coding in the human orbitofrontal cortex. J Neurosci. 2010;30(39):13095-104.

59. Eklund A, Nichols TE, Knutsson H. Cluster failure: why fMRI inferences for spatial extent have inflated false-positive rates. Proc Natl Acad Sci. 2016;113(28):7900-5.

60. Hellberg SN, Russell TI, Robinson MJF. Cued for risk: evidence for an incentive sensitization framework to explain the interplay between stress and anxiety, substance abuse, and reward uncertainty in disordered gambling behavior. Cogn Affect Behav Neurosci. 2018. https://doi.org/10.3758/s13415-018-00662-3.

61. Balodis IM, Kober H, Worhunsky PD, Stevens MC, Pearlson GD, Potenza MN. Attending to striatal ups and downs in addictions. Biol Psychiatry. 2012;72(10):e25-6.

62. Limbrick-Oldfield EH, van Holst RJ, Clark L. Fronto-striatal dysregulation in drug addiction and pathological gambling: consistent inconsistencies? Neuroimage Clin. 2013;2:385-93.

63. van Holst RJ, Veltman DJ, van den Brink W, Goudriaan AE. Right on cue? Striatal reactivity in problem gamblers. Biol Psychiatry. 2012;72(10):e23-4.

64. Leyton M, Vezina P. On cue: striatal ups and downs in addictions. Biol Psychiatry. 2012;72(10):e21-2.

65. Leyton M, Vezina P. Striatal ups and downs: their roles in vulnerability to addictions in humans. Neurosci Biobehav Rev. 2013;37(9 Pt A):1999-2014. 
66. George O, Koob GF. Individual differences in the neuropsychopathology of addiction. Dialogues Clin Neurosci. 2017;19(3):217-29.

67. Russell AMT, Hing N, Browne M, Li E, Vitartas P. Who bets on micro events (microbets) in sports? J Gambl Stud. 2018;35:20523.

68. Nyemcsok C, Thomas SL, Bestman A, Pitt H, Daube M, Cassidy $\mathrm{R}$. Young people's recall and perceptions of gambling advertising and intentions to gamble on sport. J Behav Addict. 2018;7(4): 1068-78.

69. Pitt H, Thomas SL, Bestman A, Daube M, Derevensky J. Factors that influence children's gambling attitudes and consumption intentions: lessons for gambling harm prevention research, policies and advocacy strategies. Harm Reduct J. 2017;14(1):11.

70. Pitt H, Thomas SL, Bestman A, Daube M, Derevensky J. What do children observe and learn from televised sports betting advertisements? A qualitative study among Australian children. Aust N Z J Public Health. 2017;41(6):604-10.

71. Thomas SL, Bestman A, Pitt H, Cassidy R, McCarthy S, Nyemcsok $\mathrm{C}$, et al. Young people's awareness of the timing and placement of gambling advertising on traditional and social media platforms: a study of 11-16-year-olds in Australia. Harm Reduct J. 2018;15(1):51.

72. Lopez-Gonzalez H, Estévez A, Griffiths MD. Can positive social perception and reduced stigma be a problem in sports betting? A qualitative focus group study with Spanish sports bettors undergoing treatment for gambling disorder. J Gambl Stud. 2018. https://oi.org/10.1007/s10899-018-9799-2.

73. Tibboel H, De Houwer J, Spruyt A, Brevers D, Roy E, Noël X. Heavy social drinkers score higher on implicit wanting and liking for alcohol than alcohol-dependent patients and light social drinkers. J Behav Ther Exp Psychiatry. 2015;48:185-91.

74. Breiner MJ, Stritzke WG, Lang AR. Approaching avoidance. A step essential to the understanding of craving. Alcohol Res Health. 1999;23(3):197-206

75. Smith-Hoerter K, Stasiewicz PR, Bradizza CM. Subjective reactions to alcohol cue exposure: a qualitative analysis of patients' self-reports. Psychol Addict Behav. 2004;18(4):402-6.

76. Townshend JM, Duka T. Avoidance of alcohol-related stimuli in alcohol-dependent inpatients. Alcohol Clin Exp Res. 2007;31(8): 1349-57.

77. Vollstädt-Klein S, Loeber S, von der Goltz C, Mann K, Kiefer F. Avoidance of alcohol-related stimuli increases during the early stage of abstinence in alcohol-dependent patients. Alcohol Alcohol. 2009;44(5):458-63.

78. Spruyt A, De Houwer J, Tibboel H, Verschuere B, Crombez G, Verbanck $\mathrm{P}$, et al. On the predictive validity of automatically activated approach/avoidance tendencies in abstaining alcoholdependent patients. Drug Alcohol Depend. 2013;127(1-3):81-6.

79. James RJE, O'Malley C, Tunney RJ. Understanding the psychology of mobile gambling: a behavioural synthesis. Br J Psychol. 2017;108(3):608-25

80. Cassidy R, Ovenden N. Frequency, duration and medium of advertisements for gambling and other risky products in commercial and public service broadcasts of English premier league football. SocArXiv. 2017. https://doi.org/10.31235/osf.io/f6bu8.

81. Elton-Marshall T, Leatherdale ST, Turner NE. An examination of internet and land-based gambling among adolescents in three Canadian provinces: results from the youth gambling survey (YGS). BMC Public Health. 2016;16:277.

82. Russell GEH, Williams RJ, Sanders JL. The relationship between memory associations, gambling involvement, and problem gambling. Addict Behav. 2018;92:47-52.

83. Oikonomidis A, Palomäki J, Laakasuo M. Experience and passion in poker: are there well-being implications? J Gambl Stud. 2018. https://doi.org/10.1007/s10899-018-9795-6.
84. Ryan RM, Deci EL. Self-determination theory and the facilitation of intrinsic motivation, social development, and well-being. Am Psychol. 2000;55(1):68-78.

85. Sheldon KM, Kasser T, Smith K, Share T. Personal goals and psychological growth: testing an intervention to enhance goal attainment and personality integration. J Pers. 2002;70(1):5-31.

86. Vallerand RJ, Fortier MS, Guay F. Self-determination and persistence in a real-life setting: toward a motivational model of high school dropout. J Pers Soc Psychol. 1997;72(5):1161-76.

87. Vallerand RJ, Ntoumanis N, Philippe FL, Lavigne GL, Carbonneau N, Bonneville A, et al. On passion and sports fans: a look at football. J Sports Sci. 2008;26(12):1279-93.

88. Rousseau FL, Vallerand RJ, Ratelle CF, Mageau GA, Provencher PJ. Passion and gambling: on the validation of the Gambling Passion Scale (GPS). J Gambl Stud. 2002;18(1):45-66.

89. Reith G, Dobbie F. Beginning gambling: the role of social networks and environment. Addict Res Theory. 2011;19:483-93.

90. Reith G, Dobbie F. Lost in the game: narratives of addiction and identity in recovery from problem gambling. Addict Res Theory. 2012;20:511-21.

91. Reith G, Dobbie F. Gambling careers: a longitudinal, qualitative study of gambling behaviour. Addict Res Theory. 2013;21:376-90.

92. Reith G. Addictive Consumption: Capitalism, Modernity and Excess. London: Routledge; 2018. This book explores trajectories of recovery from gambling problem, and offers insightful perspectives on how to conceptualize problematic gambling habits in modern society.

93. Dwyer B, Shapiro SL, Drayer J. Daily fantasy football and selfreported problem behavior in the United States. J Gambl Stud. 2018;34(3):689-707.

94. Dwyer B, Weiner J. Daily grind: a comparison of causality orientations, emotions, and fantasy sport participation. J Gambl Stud. 2018;34(1):1-20.

95. Gainsbury SM, King DL, Russell AMT, Delfabbro P, Derevensky J, Hing N. Exposure to and engagement with gambling marketing in social media: reported impacts on moderate-risk and problem gamblers. Psychol Addict Behav. 2016;30(2):270-6.

96.• Brevers D, Herremans SC, He Q, Vanderhasselt M-A, Petieau M, Verdonck D, et al. Facing temptation: the neural correlates of gambling availability during sports picture exposure. Cogn Affect Behav Neurosci. 2018;18(4):718-29 This fMRI study was the first to examine how the prospect of a sports betting choice impacts cue reactivity. The procedure included ecological cues, manipulated gambling reward availability/expectancy, and post-task ratings of participants' level of interest toward each cue presented during the experiment.

97. Blechert J, Klackl J, Miedl SF, Wilhelm FH. To eat or not to eat: effects of food availability on reward system activity during food picture viewing. Appetite. 2016;99:254-61.

98. Nelson SE, Edson TC, Singh P, Tom M, Martin RJ, LaPlante DA, et al. Patterns of daily fantasy sport play: tackling the issues. $\mathrm{J}$ Gambl Stud. 2019;35:181-204..

99. Bierzynska M, Bielecki M, Marchewka A, Debowska W, Duszyk A, Zajkowski W, et al. Effect of frustration on brain activation pattern in subjects with different temperament. Front Psychol. 2015;6:1989.

100. Weiss NH, Sullivan TP, Tull MT. Explicating the role of emotion dysregulation in risky behaviors: a review and synthesis of the literature with directions for future research and clinical practice. Curr Opin Psychol. 2015;3:22-9.

101. Xue G, Lu Z, Levin IP, Bechara A. The impact of prior risk experiences on subsequent risky decision-making: the role of the insula. Neuroimage. 2010;50(2):709-16.

102. Yu R, Mobbs D, Seymour B, Rowe JB, Calder AJ. The neural signature of escalating frustration in humans. Cortex. 2014;54:165-78. 
103. Gindrat A-D, Chytiris M, Balerna M, Rouiller EM, Ghosh A. Usedependent cortical processing from fingertips in touchscreen phone users. Curr Biol. 2015;25(1):109-16.

104. Gosh A, Balerna M. Neuronal control of the fingertips is socially configured in touchscreen smartphone users. bioRxiv. 2016. https://www.biorxiv.org/content/10.1101/064485v3.

105. Li Y, Wang Z, Boileau I, Dreher JC, Gelskov S, Genauck A, ... Sescousse G. Altered orbitofrontal sulcogyral patterns in gambling disorder: a multicenter study. BioRxiv. 2018 https://www. biorxiv.org/content/10.1101/439034v1 This structural brain imaging study provides a roadmap on how multicenter and data driven studies should be conducted in the clinical neuroscience of gambling disorder.
106. Kwako LE, Momenan R, Litten RZ, Koob GF, Goldman D. Addictions neuroclinical assessment: a neuroscience-based framework for addictive disorders. Biol Psychiatry. 2016;80(3):179-89.

107. Kwako LE, Momenan R, Litten RZ, Koob GF, Goldman D. Reply to: Neuroclinical assessment of addiction needs to incorporate decision-making measures and ecological validity. Biol Psychiatry. 2017;81(7):e55.

Publisher's Note Springer Nature remains neutral with regard to jurisdictional claims in published maps and institutional affiliations. 